\title{
Fluides incompressibles horizontalement visqueux
}

\author{
Marius Paicu
}

\section{Résumé}

Motivé par l'étude des fluides tournants entre deux plaques, nous considérons l'équation tridimensionnelle de Navier-Stokes incompressible avec viscosité verticale nulle.

Nous démontrons l'existence locale et l'unicité de la solution dans un espace critique (invariant par le changement d'échelle de l'équation). La solution est globale en temps si la donnée initiale est petite par rapport à la viscosité horizontale.

Nous obtenons l'unicité de la solution dans un espace plus grand que l'espace des données pour lesquelles on sait résoudre l'équation. La démonstration s'appuie sur un découpage adapté en fréquences verticales (on estime différemment la partie "basses fréquences" et la partie "hautes fréquences") et sur le contrôle précis de la régularité dans les variables horizontales.

\section{Introduction}

On considère dans la suite le système de Navier-Stokes avec viscosité verticale nulle

$$
\left(N S_{\nu_{\mathrm{v}=0}}\right)\left\{\begin{array}{l}
\partial_{t} v+v \cdot \nabla v-\nu_{h}\left(\partial_{x_{1}}^{2}+\partial_{x_{2}}^{2}\right) v=-\nabla p \quad \text { dans } \mathbb{R}_{+} \times \mathbb{R}^{3} \\
\operatorname{div} v=0 \quad \text { dans } \mathbb{R}_{+} \times \mathbb{R}^{3} \\
\left.v\right|_{t=0}=v_{0}
\end{array}\right.
$$

où $v$ est la vitesse du fluide, $p$ est un scalaire qui représente la pression et $\nu_{h}>0$, est la viscosité horizontale du fluide.

Ce système a été étudié pour la première fois dans [6]. Le choix de la viscosité verticale nulle est justifié par l'étude des modèles géophysiques de fluides. En effet, on modélise l'atmosphère et l'océan par des fluides tournants à grande vitesse. Les équations de fluides tournants sont obtenues en ajoutant à l'équation de NavierStokes tridimensionnelle, la force de Coriolis $\frac{1}{\varepsilon} v \times e_{3}$, où $e_{3}$ est le vecteur $(0,0,1)$ et $\varepsilon>0$ est le nombre de Rossby, que l'on considère comme un petit paramètre (voir [15]). La loi d'Ekman pour les fluides tournants qui évoluent entre deux plans parallèles montre que le terme de viscosité typique qu'il faut considérer est de la forme $\left(-\nu_{h} \Delta_{h} v^{\varepsilon}-\beta \varepsilon \partial_{3}^{2} v^{\varepsilon}\right)$ (voir l'article [10]). 
Dans [6], pour l'équation de Navier-Stokes sans viscosité dans la direction verticale, est étudiée dans le cas de l'espace entier, l'existence locale en temps pour des données initiales quelconques dans $H^{0, s}\left(\mathbb{R}^{3}\right)\left(s>\frac{1}{2}\right)$ et l'existence globale pour des données petites par rapport à la viscosité horizontale, dans le même espace. L'unicité est démontrée pour $s>\frac{3}{2}$ dans [6] et pour $s>\frac{1}{2}$ dans [12]. Notons que $H^{0, s}\left(\mathbb{R}^{3}\right)$ est l'espace des fonctions qui sont $L^{2}$ dans la variable horizontale et $H^{s}$ dans la variable verticale.

Il est important de penser à la propriété d'invariance de l'équation par changement d'échelle : si $v$ est une solution de l'équation $\left(N S_{\nu_{\mathrm{v}}=0}\right)$ avec donnée initiale $v_{0}$, alors $v_{\lambda}(t, x):=\lambda v\left(\lambda^{2} t, \lambda x\right)$ est encore une solution de l'équation $\left(N S_{\nu_{\mathrm{v}}=0}\right)$, avec donnée initiale $v_{0, \lambda}(x):=\lambda v_{0}(\lambda x)$. Notons que l'espace invariant par ce changement d'échelle est ici $H^{0,1 / 2}$. Une telle approche a été initiée par Fujita et Kato (voir [9]) pour l'équation traditionelle de Navier-Stokes tridimensionelle, dans le cadre de l'espace classique de Sobolev $H^{1 / 2}\left(\mathbb{R}^{3}\right)$.

Notre objectif est de reprendre les résultats démontrés dans [6] dans un espace invariant par changement d'échelle et "proche" de $H^{0,1 / 2}$.

Remarquons que l'absence de viscosité dans une direction empêche d'utiliser une méthode "à la Leray" pour définir des solutions faibles dans l'espace d'énergie $L^{2}\left(\mathbb{R}^{3}\right)$.

Nous allons travailler avec la donnée initiale dans un espace mixte, de type "Besov-Sobolev" anisotrope, qui est un espace critique dans le sens où il est invariant par rapport au changement d'échelle de l'équation de Navier-Stokes. Cet espace contient les espaces de Sobolev anisotropes $H^{0, s}$ avec $s>1 / 2$.

\section{Notations et énoncés des résultats}

Avant d'énoncer les résultats, définissons les espaces avec lesquels on travaille.

L'espace de Sobolev anisotrope $H^{0, s}$ est défini par

$$
H^{0, s}\left(\mathbb{R}^{3}\right)=\left\{u \in \mathcal{S}^{\prime}\left(\mathbb{R}^{3}\right) \mid \hat{u} \in L_{l o c}^{1}\left(\mathbb{R}^{3}\right) \text { et }\|u\|_{H^{0, s}}^{2}:=\int_{\mathbb{R}^{3}}\left(1+\left|\xi_{3}\right|\right)^{2 s}|\hat{u}(\xi)|^{2} d \xi<\infty\right\} .
$$

Pour atteindre le cas limite $s=1 / 2$, nous allons remplacer l'espace de Sobolev anisotrope $H^{0, \frac{1}{2}}$ par l'espace mixte "Besov - Sobolev" anisotrope $\mathcal{B}^{0,1 / 2}$. Introduisons cet espace par l'adhérence de fonctions régulières pour la norme :

$$
\|u\|_{\mathcal{B}^{0,1 / 2}}=\sum_{q \in \mathbb{Z}}\left(\int_{\xi_{h}} \int_{2^{q-1}<\left|\xi_{3}\right|<2^{q}}\left|\xi_{3}\right||\hat{u}(\xi)|^{2} d \xi\right)^{1 / 2}<\infty .
$$

Il est facile de voir que cette norme est équivalente à la norme suivante

$$
\|u\|_{\mathcal{B}^{0,1 / 2}}=\sum_{q \in \mathbb{Z}} 2^{q / 2}\left\|\triangle_{q}^{\mathrm{v}} u\right\|_{L^{2}\left(\mathbb{R}^{3}\right)}<\infty
$$

où $\triangle_{q}^{\mathrm{v}}$ désigne les opérateurs de localisation en fréquences verticales (voir la définition dans la section 2). 
Cet espace contient les espaces $H^{0, s}(s>1 / 2)$ et il est un peu plus petit que $H^{0,1 / 2}$ mais il a les mêmes proprietés d'invariance par changement d'échelle. La raison pour laquelle on utilise l'espace $\mathcal{B}^{0,1 / 2}$ au lieu de $H^{0,1 / 2}$ est donnée par le fait que cet espace est inclus dans $L^{\infty}\left(\mathbb{R}_{x_{3}} ; L^{2}\left(\mathbb{R}_{x_{1}} \times \mathbb{R}_{x_{2}}\right)\right)=: L_{\mathrm{v}}^{\infty} L_{h}^{2}$ et l'inclusion de $H^{0, s}$ (avec $s>1 / 2$ ) dans l'espace $L_{\mathrm{v}}^{\infty}\left(L_{h}^{2}\right)$ a joué un rôle important dans les démonstrations données dans [6].

Le théorème principal de l'article est un résultat d'existence locale et d'unicité des solutions pour l'équation de Navier-Stokes avec viscosité verticale nulle. Nous allons démontrer l'existence et l'unicité de la solution dans l'espace $\mathcal{B}^{0,1 / 2}$ (voir le théorème 1). Nous démontrons également l'unicité de la solution de l'équation $\left(N S_{\nu_{\mathrm{v}}=0}\right)$ lorsque la donnée initiale est dans l'espace non-homogène $H^{0,1 / 2}$, espace qui n'est pas comparable avec $C^{-1}$ (voir le théorème 2). L'intérêt de cet espace est le fait qu'on connait l'unicité de la solution de l'équation classique de Navier-Stokes tridimensionelle dans $C^{-1}$. Ce résultat a été démontré par J.-Y. Chemin dans [4].

Théorème 1 Soit $v_{0} \in \mathcal{B}^{0,1 / 2}\left(\mathbb{R}^{3}\right)$ un champ de vecteurs de divergence nulle. Alors, il existe un temps $T>0$ tel que l'équation $\left(N S_{\nu_{\mathrm{v}}=0}\right)$ a une unique solution

$$
v \in C_{b}\left([0, T], \mathcal{B}^{0,1 / 2}\right) \text { avec } \nabla_{h} v \in L^{2}\left([0, T], \mathcal{B}^{0,1 / 2}\right) .
$$

De plus, il existe une constante c telle que si

$$
\left\|v_{0}\right\|_{\mathcal{B}^{0,1 / 2}} \leq c \nu_{h}
$$

alors, l'équation $\left(N S_{\nu_{\mathrm{v}}=0}\right)$ admet une unique solution globale en temps

$$
v \in C_{b}\left(\mathbb{R}_{+}, \mathcal{B}^{0,1 / 2}\right) \text { avec } \nabla_{h} v \in L^{2}\left(\mathbb{R}_{+}, \mathcal{B}^{0,1 / 2}\right) .
$$

Soit $T^{*}$ le temps de vie maximal pour $v$. On a

$$
\text { Si } T^{*}<\infty \text { alors }\|v\|_{\widetilde{T_{T^{*}}^{\infty}}\left(\mathcal{B}^{0,1 / 2}\right)}=+\infty \text {. }
$$

Le théorème suivant est un résultat d'unicité dans un espace qui n'est pas inclus dans $C^{-1}$. Notons par $H^{1,1 / 2}$ l'espace des fonctions dont le gradient dans la variable horizontale appartient à l'espace $H^{0,1 / 2}$.

Théorème 2 L'équation $\left(N S_{\nu_{\mathrm{v}}=0}\right)$ admet au plus une solution dans l'espace

$$
L_{T}^{\infty}\left(H^{0,1 / 2}\right) \cap L_{T}^{2}\left(H^{1,1 / 2}\right) .
$$

Remarque 1.1 Notons que ce théorème est nouveau aussi pour l'équation de NavierStokes classique.

\section{Préliminaires}

Tout d'abord, rappelons la définition des espaces de Lebesgue anisotropes. On désigne par $L_{h}^{p}\left(L_{\mathrm{v}}^{q}\right)$ l'espace $L^{p}\left(\mathbb{R}_{x_{1}} \times \mathbb{R}_{x_{2}} ; L^{q}\left(\mathbb{R}_{x_{3}}\right)\right)$ qui est muni de la norme : 


$$
\begin{aligned}
\|f\|_{L_{h}^{p}\left(L_{\mathrm{v}}^{q}\right)}:=\|\| f\left(x_{h}, \cdot\right)\left\|_{L^{q}\left(\mathbb{R}_{x_{3}}\right)}\right\|_{L^{p}\left(\mathbb{R}_{x_{1}} \times \mathbb{R}_{x_{2}}\right)} & =\left(\int_{\mathbb{R}_{x_{1}} \times \mathbb{R}_{x_{2}}}\left(\int_{\mathbb{R}_{x_{3}}}\left|f\left(x_{h}, x_{3}\right)\right|^{q} d x_{3}\right)^{p / q} d x_{h}\right)^{1 / p} .
\end{aligned}
$$

De la même manière, on note par $L_{\mathrm{v}}^{q}\left(L_{h}^{p}\right)$ l'espace $L^{q}\left(\mathbb{R}_{x_{3}} ; L^{p}\left(\mathbb{R}_{x_{1}} \times \mathbb{R}_{x_{2}}\right)\right)$ avec la norme associée $\|f\|_{L_{\mathrm{v}}^{q}\left(L_{h}^{p}\right)}:=\|\| f\left(\cdot, x_{3}\right)\left\|_{L^{p}\left(\mathbb{R}_{x_{1}} \times \mathbb{R}_{x_{2}}\right)}\right\|_{L^{q}\left(\mathbb{R}_{x_{3}}\right)}$.

C'est bien connu que l'ordre d'intégration est important dans le sens décrit par le lemme ci-après :

Lemme 2.1 Soient $1 \leq p \leq q$ et $f: X_{1} \times X_{2} \rightarrow \mathbb{R}$ une fonction appartenant $\grave{a}$ $L^{p}\left(X_{1} ; L^{q}\left(X_{2}\right)\right)$, où $\left(X_{1} ; d \mu_{1}\right)$ et $\left(X_{2} ; d \mu_{2}\right)$ sont des espaces mesurables. Alors $f \in$ $L^{q}\left(X_{2} ; L^{p}\left(X_{1}\right)\right)$ et on a l'inégalité

$$
\|f\|_{L^{q}\left(X_{2} ; L^{p}\left(X_{1}\right)\right)} \leq\|f\|_{L^{p}\left(X_{1} ; L^{q}\left(X_{2}\right)\right)} .
$$

L'outil de base dans les démonstrations sera la théorie de Littlewood-Paley anisotrope qui consiste à faire un découpage dyadique dans les fréquences verticales.

Introduisons les opérateurs de troncature en fréquences verticales comme suit :

$$
\triangle_{q}^{\mathrm{v}} u=\mathcal{F}^{-1}\left(\varphi\left(\frac{\left|\xi_{3}\right|}{2^{q}}\right) \mathcal{F} u(\xi)\right),
$$

où on a noté par $\mathcal{F}$ la transformation de Fourier et $u \in \mathcal{S}^{\prime}\left(\mathbb{R}^{3}\right)$.

Les fonctions $\varphi$ et $\chi$ représentent une partition de l'unité dans $\mathbb{R}$, elles sont régulières et on a

$$
\text { supp } \chi \subset B(0,4 / 3), \text { supp } \varphi \subset \mathcal{C}(3 / 4,8 / 3) \text {, }
$$

et

$$
\chi(t)+\sum_{q \geq 0} \varphi\left(2^{-q} t\right)=1 .
$$

Définissons aussi l'opérateur :

$$
S_{q}^{\mathrm{v}} u=\sum_{q^{\prime} \leq q-1} \triangle_{q^{\prime}}^{\mathrm{v}} u
$$

Nous utilisons constamment le lemme de Bernstein anisotrope suivant.

Lemme 2.2 Soit $u$ une fonction avec supp $\mathcal{F}^{\mathrm{v}} u \subset \mathbb{R}_{h}^{2} \times 2^{q} \mathcal{C}$, où l'on a noté par $\mathcal{F}^{\mathrm{v}}$ la transformation de Fourier dans la variable verticale et où $\mathcal{C}$ est une couronne dyadique. Soient $p \geq 1$ et $r \geq r^{\prime} \geq 1$, nombres réels. On a:

$$
\begin{gathered}
2^{q k} C^{-k}\|u\|_{L_{h}^{p}\left(L_{\mathrm{v}}^{r}\right)} \leq\left\|\partial_{x_{3}}^{k} u\right\|_{L_{h}^{p}\left(L_{\mathrm{v}}^{r}\right)} \leq 2^{q k} C^{k}\|u\|_{L_{h}^{p}\left(L_{\mathrm{v}}^{r}\right)} ; \\
2^{q k} C^{-k}\|u\|_{L_{\mathrm{v}}^{r}\left(L_{h}^{p}\right)} \leq\left\|\partial_{x_{3}}^{k} u\right\|_{L_{\mathrm{v}}^{r}\left(L_{h}^{p}\right)} \leq 2^{q k} C^{k}\|u\|_{L_{\mathrm{v}}^{r}\left(L_{h}^{p}\right)} ; \\
\|u\|_{L_{h}^{p}\left(L_{\mathrm{v}}^{r}\right)} \leq C 2^{q\left(\frac{1}{r^{\prime}}-\frac{1}{r}\right)}\|u\|_{L_{h}^{p}\left(L_{\mathrm{v}}^{r^{\prime}}\right)} \\
\|u\|_{L_{\mathrm{v}}^{r}\left(L_{h}^{p}\right)} \leq C 2^{q\left(\frac{1}{r^{\prime}}-\frac{1}{r}\right)}\|u\|_{L_{\mathrm{v}}^{r^{\prime}}\left(L_{h}^{p}\right)} .
\end{gathered}
$$


Nous allons utiliser aussi l'inégalité de "Gagliardo-Nirenberg" anisotrope suivante.

Lemme 2.3 Pour toute fonction u, nous avons les estimations suivantes

$$
\|u\|_{L^{2}\left(\mathbb{R}_{x_{3}} ; L^{4}\left(\mathbb{R}_{x_{1}} \times \mathbb{R}_{x_{2}}\right)\right)} \leq C\|u\|_{L^{2}\left(\mathbb{R}^{3}\right)}^{1 / 2}\left\|\nabla_{h} u\right\|_{L^{2}\left(\mathbb{R}^{3}\right)}^{1 / 2} .
$$

\section{Calcul paradifférentiel anisotrope.}

La décomposition dyadique est utile aussi dans l'étude du produit de deux distributions. Formellement, on peut écrire, pour deux distributions $u$ et $v$,

$$
u=\sum_{q \in \mathbb{Z}} \triangle_{q}^{\mathrm{v}} u \quad ; \quad v=\sum_{q \in \mathbb{Z}} \triangle_{q}^{\mathrm{v}} v \quad \text { et } \quad u \cdot v=\sum_{q \in \mathbb{Z}, q^{\prime} \in \mathbb{Z}} \triangle_{q}^{\mathrm{v}} u \cdot \triangle_{q^{\prime}}^{\mathrm{v}} v .
$$

On va séparer cette somme en trois sommes, l'une où les fréquences verticales de $u$ sont petites devant les fréquences verticales de $v$, une autre où les fréquences verticales de $v$ sont petites devant les fréquences verticales de $u$, et la dernière somme, où les fréquences de $u$ et de $v$ sont comparables. Les premières deux sommes sont les paraproduits et la dernière est le reste. Cette décomposition s'appelle la décomposition de Bony dans la variable verticale (voir [1] respectivement [6] et [11] pour le cas anisotrope).

Nous allons noter :

$$
T_{u} v=\sum_{q^{\prime} \leq q-2} \triangle_{q^{\prime}}^{\mathrm{v}} u \cdot \triangle_{q}^{\mathrm{v}} v=\sum_{q} S_{q-1}^{\mathrm{v}} u \cdot \triangle_{q}^{\mathrm{v}} v
$$

et

$$
R(u, v)=\sum_{q} \sum_{i \in\{0, \pm 1\}} \triangle_{q}^{\mathrm{v}} u \triangle_{q+i}^{\mathrm{v}} v .
$$

Remarquons qu'on a des bonnes propriétés de quasi-orthogonalité dans le sens

$$
\begin{array}{lllll}
\triangle_{q}^{\mathrm{v}}\left(S_{q^{\prime}-1}^{\mathrm{v}} u \triangle_{q^{\prime}}^{\mathrm{v}} v\right) & =0 & \text { pour } & \left|q-q^{\prime}\right| \geq 5 & \text { et aussi } \\
\triangle_{q}^{\mathrm{v}}\left(S_{q^{\prime}+1}^{\mathrm{v}} u \triangle_{q^{\prime}}^{\mathrm{v}} v\right) & =0 & \text { pour } & q^{\prime} \leq q-4 .
\end{array}
$$

Pour alléger les démonstrations qui s'appuient sur le calcul paradifférentiel, nous allons noter par $a_{q}$ une suite génerique qui est de racine carrée sommable, c'est-àdire, $a_{q} \geq 0$ et

$$
\sum_{q \in \mathbb{Z}} \sqrt{a}_{q} \leq 1
$$

On va désigner par $b_{q} \geq 0$ une suite générique sommable et par $c_{q}$ une suite de carré sommable telle que

$$
\sum_{q \in \mathbb{Z}} b_{q} \leq 1 \quad \text { et } \quad \sum_{q \in \mathbb{Z}} c_{q}^{2} \leq 1
$$

Notons ici, que l'appartenance d'une fonction $u$ à l'espace $\mathcal{B}^{0,1 / 2}$ se traduit par l'existence d'une suite $b_{q}$, telle que

$$
\left\|\triangle_{q}^{\mathrm{v}} u\right\|_{L^{2}} \leq C 2^{-q / 2} b_{q}\|u\|_{\mathcal{B}^{0,1 / 2}} .
$$


Dans le même esprit, notons que $u$ appartient à l'espace de Sobolev $H^{0, s}$ si et seulement si

$$
\left\|\triangle_{q}^{\mathrm{v}} u\right\|_{L^{2}} \leq C 2^{-q s} c_{q}\|u\|_{H^{0, s}}
$$

Notons par $\widetilde{L_{T}^{p}}\left(\mathcal{B}^{0,1 / 2}\right)$ avec $p \geq 1$, l'espace défini par la norme :

$$
\|u\|_{\widetilde{L_{T}^{p}}\left(\mathcal{B}^{0,1 / 2}\right)}=\sum_{q \in \mathbb{Z}} 2^{q / 2}\left\|\triangle_{q}^{\mathrm{v}} u\right\|_{L^{p}\left([0, T] ; L^{2}\left(\mathbb{R}^{3}\right)\right)}<+\infty
$$

La définition de ces espaces s'inspire de l'article [5].

\section{Estimations d'énergie et démonstration du théorème}

\subsection{Estimations d'énergie pour l'équation de Navier-Stokes}

L'estimation d'énergie que nous allons utiliser est semblable à l'estimation d'énergie donnée dans [6], mais dans le cadre des espaces $\widetilde{L_{T}^{p}}\left(\mathcal{B}^{0,1 / 2}\right)$.

Lemme 3.1 (estimation pour l'existence locale) Soient $u$ et $v$ deux champs de vecteurs dépendant du temps sur $\mathbb{R}^{3}$, avec $u(t)$ de divergence nulle pour tout $t \in[0, T](T>0)$. Il existe une suite de nombres réels $a_{q} \geq 0$, avec $\sum_{q \in \mathbb{Z}} a_{q}^{1 / 2} \leq 1$, qui dépendent de $u, v$ et $T$, telle que

$$
\begin{aligned}
& \int_{0}^{T}\left|\left(\triangle_{q}^{\mathrm{v}}(u \cdot \nabla v) \mid \triangle_{q}^{\mathrm{v}} v\right)_{L^{2}}\right| d t
\end{aligned}
$$

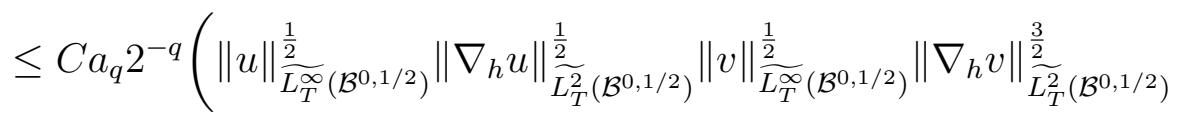

$$
\begin{aligned}
& \left.+\left\|\nabla_{h} u\right\|_{\widetilde{L_{T}^{2}\left(\mathcal{B}^{0,1 / 2}\right)}}\|v\|_{\widetilde{L_{T}^{\infty}\left(\mathcal{B}^{0,1 / 2}\right)}}\left\|\nabla_{h} v\right\|_{\widetilde{L_{T}^{2}}\left(\mathcal{B}^{0,1 / 2}\right)}\right)
\end{aligned}
$$

où $C>0$ est une constante universelle.

En particulier, on utilisera cette estimation dans le cas où $u=v$, dans la démonstration de l'existence globale. Nous avons ainsi le corollaire suivant :

Corollaire 1 (estimation pour l'existence globale) Il existe une constante $C$ telle que, pour tout champ de vecteur $v$ de divergence nulle, on a

$$
\int_{0}^{T}\left|\left(\triangle_{q}(v \nabla v)(t) \mid \triangle_{q}^{\mathrm{v}} v(t)\right)_{L^{2}}\right| d t \leq C 2^{-q} a_{q}\left\|\nabla_{h} v\right\|_{\widetilde{L_{T}^{2}\left(\mathcal{B}^{0,1 / 2}\right)}}^{2}\|v\|_{\widetilde{L_{T}^{\infty}\left(\mathcal{B}^{0,1 / 2}\right)}}
$$

avec $\sum_{q \in \mathbb{Z}} a_{q}^{1 / 2} \leq 1$

On donne à présent l'estimation nécessaire pour démontrer l'unicité. 
Lemme 3.2 (estimation pour l'unicité) Soient u et $v$ deux champs de divergence nulle, qui appartiennent à l'espace $L_{T}^{\infty}\left(H^{0,1 / 2}\right)$ et tels que $\nabla_{h} u$ et $\nabla_{h} v$ appartiennent à l'espace $L_{T}^{2}\left(H^{0,1 / 2}\right)$. Soit $w$ dans $L_{T}^{\infty}\left(H^{0,1 / 2}\right)$ avec $\nabla_{h} w$ appartenant à l'espace $L_{T}^{2}\left(H^{0,1 / 2}\right)$ vérifiant l'équation

$$
\left\{\begin{array}{l}
\partial_{t} w+u \cdot \nabla w+w \cdot \nabla v-\nu_{h} \Delta_{h} w=-\nabla p \\
\operatorname{div} w=0
\end{array}\right.
$$

Alors, pour tout $0<t<T$, si $\|w(t)\|_{H^{0,-1 / 2}} \leq e^{-1}$, on a l'estimation

$$
\frac{d}{d t}\|w(t)\|_{H^{0,-1 / 2}}^{2} \leq C f(t)\|w(t)\|_{H^{0,-1 / 2}}^{2}\left(1-\ln \|w(t)\|_{H^{0,-1 / 2}}^{2}\right) \ln \left(1-\ln \|w(t)\|_{H^{0,-1 / 2}}^{2}\right)
$$

où $f(t)$ est la fonction localement intégrable en temps donnée par

$$
\begin{aligned}
f(t)=(1+\| u(t) & \left.\left\|_{H^{0,1 / 2}}^{2}+\right\| v(t)\left\|_{H^{0,1 / 2}}^{2}+\right\| w(t) \|_{H^{0,1 / 2}}^{2}\right) \\
& \times\left(1+\left\|\nabla_{h} u(t)\right\|_{H^{0,1 / 2}}^{2}+\left\|\nabla_{h} v(t)\right\|_{H^{0,1 / 2}}^{2}+\left\|\nabla_{h} w(t)\right\|_{H^{0,1 / 2}}^{2}\right) .
\end{aligned}
$$

\subsection{Démonstration du théorème 1}

\section{Existence globale pour des données petites}

Nous allons démontrer seulement l'existence globale pour des données initiales petites. La démonstration s'appuiera sur le corollaire 1 . On va utiliser la méthode de Friedrichs, qui consiste en l'approximation du système $\left(N S_{\nu_{\mathrm{v}}=0}\right)$ par une troncature dans l'espace des fréquences. Définissons alors les opérateurs suivants

$$
\begin{aligned}
J_{n} u & =\mathcal{F}^{-1}\left(\mathbf{1}_{B(0, n)} \mathcal{F} u(\xi)\right) \\
J_{n}^{\mathrm{v}} u & =\mathcal{F}^{-1}\left(\mathbf{1}_{\left\{\xi|| \xi_{3} \mid \leq n\right\}} \mathcal{F} u(\xi)\right) \quad \text { et } \\
\tilde{J}_{n} u & =\left(J_{n}-J_{\frac{1}{n}}^{\mathrm{v}}\right) u .
\end{aligned}
$$

où on a noté par $\mathcal{F}$ la transformation de Fourier sur $\mathbb{R}^{3}$. Considérons le système approximant suivant

$$
\left(N S_{\nu_{\mathrm{v}=0}}^{n}\right)\left\{\begin{array}{l}
\partial_{t} v_{n}+\tilde{J}_{n}\left(\tilde{J}_{n} v_{n} \cdot \nabla \tilde{J}_{n} v_{n}\right)-\nu_{h} \tilde{J}_{n} \Delta_{h} v_{n}=\tilde{J}_{n} \nabla \sum_{i, j} \partial_{i} \partial_{j} \Delta^{-1}\left(\tilde{J}_{n} v_{n}^{i} \tilde{J}_{n} v_{n}^{j}\right) \\
\operatorname{div} v_{n}=0 \\
\left.v_{n}\right|_{t=0}=\tilde{J}_{n} v_{0} .
\end{array}\right.
$$

Comme il est clair que les opérateurs bilinéaires sont continus de $L^{2} \times L^{2}$ dans $L^{2}$ et comme la donnée initiale $v_{n}(0)=\tilde{J}_{n} v_{0}$ appartient à $L^{2}$ lorsque $v_{0}$ est dans l'espace homogène $\mathcal{B}^{0,1 / 2}$, alors le système $\left(N S_{\nu_{\mathrm{v}}=0}^{n}\right)$ devient une équation différentielle dans $L^{2}$. Grâce au théorème de Cauchy-Lipschitz, il existe une unique solution locale en temps à valeurs dans $L^{2}$. Notons par $T_{n}$ le temps de vie maximal pour l'unique solution $v_{n}$ du système $\left(N S_{\nu_{\mathrm{v}}=0}^{n}\right)$. Comme $\tilde{J}_{n}^{2}=\tilde{J}_{n}$, on a évidemment que $\tilde{J}_{n} v_{n}$ est aussi une solution pour $\left(N S_{\nu_{\mathrm{v}}=0}^{n}\right)$, alors, l'unicité de la solution implique que 
$v_{n}=\tilde{J}_{n} v_{n}$. Donc, on a que $v_{n}$ est solution du système suivant, qu'on va noter toujours par $\left(N S_{\nu_{\mathrm{v}}=0}^{n}\right)$

$$
\left(N S_{\nu_{\mathrm{v}=0}}^{n}\right)\left\{\begin{array}{l}
\partial_{t} v_{n}+\tilde{J}_{n}\left(v_{n} \cdot \nabla v_{n}\right)-\nu_{h}\left(\partial_{x_{1}}^{2}+\partial_{x_{2}}^{2}\right) v_{n}=\tilde{J}_{n} \nabla \sum_{i, j} \partial_{i} \partial_{j} \Delta^{-1}\left(v_{n}^{i} v_{n}^{j}\right) \\
\operatorname{div} v_{n}=0 \\
\left.v_{n}\right|_{t=0}=\tilde{J}_{n} v_{0} .
\end{array}\right.
$$

Puisque $\left(\tilde{J}_{n}\left(v_{n} \nabla v_{n}\right) \mid v_{n}\right)_{L^{2}}=0$, l'estimation d'énergie dans $L^{2}$ nous donne

$$
\frac{1}{2} \frac{d}{d t}\left\|v_{n}(t)\right\|_{L^{2}}^{2}+\nu_{h}\left\|\nabla_{h} v_{n}(t)\right\|_{L^{2}}^{2}=0
$$

Donc, $v_{n}(t)$ reste bornée sur l'intervalle maximal d'existence $\left[0, T_{n}\right)$, d'où on déduit facilement que $T_{n}=+\infty$.

Afin d'alléger les notations, on "oublie" dorénavant l'indice $n$. On va appliquer l'opérateur $\triangle_{q}^{\mathrm{v}}$ à l'équation approximante $\left(N S_{\nu_{\mathrm{v}=0}}^{n}\right)$ et on va faire les estimations d'énergie dans $L^{2}$ sur chaque morceau $\triangle_{q}^{\mathrm{v}} v$, et ensuite, on va recoller les estimations. Nous obtenons ainsi :

$$
\begin{aligned}
\left\|\triangle_{q}^{\mathrm{v}} v(t)\right\|_{L^{2}}^{2}+2 \nu_{h} \int_{0}^{t} \| \nabla_{h} & \triangle_{q}^{\mathrm{v}} v(\tau) \|_{L^{2}}^{2} d \tau \\
& \leq\left\|\triangle_{q}^{\mathrm{v}} v_{0}\right\|_{L^{2}}^{2}+2 \int_{0}^{t}\left|\left(\triangle_{q}^{\mathrm{v}}(v \cdot \nabla v)(\tau) \mid \triangle_{q}^{\mathrm{v}} v(\tau)\right)_{L^{2}}\right| d \tau
\end{aligned}
$$

En prenant le supremum en $t \in[0, T]$, on a

$$
\begin{aligned}
\left\|\triangle_{q}^{\mathrm{v}} v\right\|_{L_{T}^{\infty}\left(L^{2}\right)}^{2}+2 \nu_{h} \| \nabla_{h} \triangle_{q}^{\mathrm{v}} v & \|_{L_{T}^{2}\left(L^{2}\right)}^{2} \\
& \leq\left\|\triangle_{q}^{\mathrm{v}} v_{0}\right\|_{L^{2}}^{2}+2 \int_{0}^{T}\left|\left(\triangle_{q}^{\mathrm{v}}(v \cdot \nabla v)(\tau) \mid \triangle_{q}^{\mathrm{v}} v(\tau)\right)_{L^{2}}\right| d \tau
\end{aligned}
$$

Nous allons utiliser maintenant le corollaire 1 pour obtenir que

$$
\begin{aligned}
& \left\|\triangle_{q}^{\mathrm{v}} v\right\|_{L_{T}^{\infty}\left(L^{2}\right)}^{2}+2 \nu_{h}\left\|\nabla_{h} \triangle_{q}^{\mathrm{v}} v\right\|_{L_{T}^{2}\left(L^{2}\right)}^{2} \\
& \quad \leq\left\|\triangle_{q}^{\mathrm{v}} v_{0}\right\|_{L^{2}}^{2}+2^{-q} a_{q} C\|v\|_{\widetilde{L_{T}^{\infty}\left(\mathcal{B}^{0,1 / 2}\right)}}\left\|\nabla_{h} v\right\|_{\widetilde{L_{T}^{2}\left(\mathcal{B}^{0,1 / 2}\right)}}^{2}
\end{aligned}
$$

avec $\sum_{q} \sqrt{a_{q}} \leq 1$. D'où, par l'équivalence entre $a^{2}+b^{2}$ et $(a+b)^{2}$ quand $a$ et $b$ sont des nombres réels positifs, il vient

$$
\begin{aligned}
2^{q / 2}\left\|\triangle_{q}^{\mathrm{v}} v\right\|_{L_{T}^{\infty}\left(L^{2}\right)}+ & \sqrt{2} \nu_{h}^{1 / 2} 2^{q / 2}\left\|\nabla_{h} \triangle_{q}^{\mathrm{v}} v\right\|_{L_{T}^{2}\left(L^{2}\right)} \\
& \leq 2^{q / 2} \sqrt{2}\left\|\triangle_{q}^{\mathrm{v}} v_{0}\right\|_{L^{2}}+a_{q}^{1 / 2} \sqrt{2} C^{1 / 2}\|v\|_{\widetilde{L_{T}^{\infty}}\left(\mathcal{B}^{0,1 / 2}\right)}^{1 / 2}\left\|\nabla_{h} v\right\|_{\widetilde{L_{T}^{2}}\left(\mathcal{B}^{0,1 / 2}\right)}
\end{aligned}
$$

Par sommation en $q$, nous obtenons

$$
\begin{aligned}
\|v\|_{\widetilde{L_{T}^{\infty}}\left(\mathcal{B}^{0,1 / 2}\right)}+\sqrt{2} \nu_{h}^{1 / 2} & \left\|\nabla_{h} v\right\|_{\widetilde{L_{T}^{2}}\left(\mathcal{B}^{0,1 / 2}\right)} \\
& \leq \sqrt{2}\left\|v_{0}\right\|_{\mathcal{B}^{0,1 / 2}}+C^{1 / 2} \sqrt{2}\|v\|_{\widetilde{L_{T}^{\infty}\left(\mathcal{B}^{0,1 / 2}\right)}}^{1 / 2}\left\|\nabla_{h} v\right\|_{\widetilde{L_{T}^{2}\left(\mathcal{B}^{0,1 / 2}\right)}} .
\end{aligned}
$$


On peut alors en déduire l'existence globale lorsque la donnée initiale est petite par rapport à la viscosité horizontale. En effet, soit $c$ assez petit et $v_{0} \in \mathcal{B}^{0,1 / 2}$ un champ de divergence nulle, tel que $\left\|v_{0}\right\|_{\mathcal{B}^{0,1 / 2}}<c \nu_{h}$ avec $c<\frac{1}{8 C}$. Soit $v_{n}$ la solution régulière de l'équation approximante de l'équation de Navier-Stokes anisotrope, avec la donnée initiale régularisée $\left(\left.v_{n}\right|_{t=0}=\tilde{J}_{n} v_{0}\right)$. Soit alors $T_{n}^{*}$ le temps maximal sur lequel on a $\left\|v_{n}\right\|_{\widetilde{L_{T}^{\infty}\left(\mathcal{B}^{0,1 / 2}\right)}} \leq 2 c \nu_{h}$ pour tout $0<T<T_{n}^{*}$. À l'aide de l'estimation (10), on obtient $\left\|v_{n}\right\|_{\overparen{L_{T}^{\infty}\left(\mathcal{B}^{0,1 / 2}\right)}} \leq 2\left\|v_{0}\right\|_{\mathcal{B}^{0,1 / 2}}<2 c \nu_{h}$ pour tout temps $T$ avec $0<T<T_{n}^{*}$.

$\mathrm{Vu}$ que la fonction $v_{n}$ est régulière en temps à valeurs dans $\mathcal{B}^{0,1 / 2}$, nous obtenons $T_{n}^{*}=+\infty$, et on a que la suite des solutions globales $v_{n}$ est bornée dans l'espace

$$
v_{n} \in \widetilde{L^{\infty}}\left(\mathbb{R}_{+}, \mathcal{B}^{0,1 / 2}\right) \quad \text { avec } \quad \nabla_{h} v_{n} \in \widetilde{L^{2}}\left(\mathbb{R}_{+}, \mathcal{B}^{0,1 / 2}\right) .
$$

En particulier, on obtient que $\left(v_{n}\right)_{n \in \mathbb{N}}$ est une suite bornée dans l'espace $L^{\infty}\left(\mathbb{R}_{+} ; L_{\text {loc }}^{2}\right)$ et en utilisant l'équation vérifiée par $v_{n}$, on obtient que

$$
\left(\partial_{t} v_{n}\right)_{n \in \mathbb{N}} \text { est suite bornée dans } L^{\infty}\left(\mathbb{R}_{+} ; H_{l o c}^{-N}\right)
$$

avec $N$ suffisamment grand. Comme conséquence du théorème d'Arzela-Ascoli, on a qu'il existe une sous-suite de $v_{n}$ qu'on va persister à noter $v_{n}$, tel que $v_{n} \longrightarrow v$ dans $L_{l o c}^{\infty}\left(\mathbb{R}_{+} ; H_{l o c}^{-N}\right)$. Parce que $v_{n}$ est une suite bornée dans $L^{\infty}\left(\mathbb{R}_{+} ; L_{\text {loc }}^{2}\right)$, on obtient par interpolation que

$$
v_{n} \longrightarrow v \text { dans } L_{l o c}^{\infty}\left(\mathbb{R}_{+} ; H_{l o c}^{-\sigma}\right) \text { pour tout } \sigma>0 .
$$

Vu que $v_{n}$ est une suite bornée dans $L_{\text {loc }}^{2}\left(\mathbb{R}_{+} ; H_{\text {loc }}^{\varepsilon}\right)$ pour tout $\varepsilon<1 / 2$, on obtient comme conséquence des lois de produits dans les espaces de Sobolev classiques que

$$
v_{n} \otimes v_{n} \longrightarrow v \otimes v \quad \text { dans } \quad L_{l o c}^{2}\left(H_{l o c}^{\varepsilon-\sigma-3 / 2}\right) \quad \text { lorsque } \sigma<\varepsilon .
$$

En particulier

$$
v_{n} \otimes v_{n} \longrightarrow v \otimes v \quad \text { dans } \quad \mathcal{D}^{\prime} .
$$

Par passage à la limite dans l'équation approximante $\left(N S_{\nu_{\mathrm{v}}=0}^{n}\right)$ on obtient une solution globale du système $\left(N S_{\nu_{\mathrm{v}}=0}\right)$.

\section{Démonstration du théorème 2}

\section{Démonstration de l'unicité dans $H^{0,1 / 2}$}

Avant de commencer la démonstration de l'unicité, qui est une application du lemme 3.2, donnons quelques commentaires sur les difficultés qu'on rencontre lorsque l'on tente de démontrer l'unicité de la solution pour l'équation $\left(N S_{\nu_{\mathrm{v}}=0}\right)$ et sur les méthodes utilisées pour obtenir le lemme 3.2.

Soient $u$ et $v$ deux fonctions de $L_{T}^{\infty}\left(H^{0,1 / 2}\right)$ telles que $\nabla_{h} u$ et $\nabla_{h} v$ appartiennent à $L_{T}^{2}\left(H^{0,1 / 2}\right)$. On suppose que $u$ et $v$ sont solutions de l'équation $\left(N S_{h}\right)$ pour la même donnée initiale dans $H^{0,1 / 2}$. On note $w=v-u$ et l'objectif est de démontrer que $w=0$ dans $\mathcal{D}^{\prime}\left(\mathbb{R}_{+}^{*} \times \mathcal{T}^{d}\right)$. L'équation vérifiée par $w$ donne comme termes nonlinéaires $v \cdot \nabla w$ et $w \cdot \nabla u$. Les termes $v_{h} \cdot \nabla_{h} w$ et $w_{h} \cdot \nabla_{h} u$ peuvent être estimés à 
l'aide des lois de produits (ce qui revient en fait à la décomposition de Bony dans la variable verticale). Le terme $v_{3} \cdot \partial_{3} w$ sera estimé grâce aux méthodes introduites dans [6].

Reste à estimer le terme $w_{3} \cdot \partial_{3} u$. Vu la régularité dans la troisième variable, il est le produit entre un élément de $H_{\mathrm{v}}^{1 / 2}$ et un élément de $H_{\mathrm{v}}^{-1 / 2}$, ce qui donne un élément de $B_{2, \infty}^{-1 / 2}$. Cela justifie une perte d'une dérivée en variable verticale dans les estimations sur $w$ (voir aussi [12]). On peut ainsi envisager de faire les estimations sur $w$ dans des espaces qui sont $\mathcal{B}_{2, \infty}^{-1 / 2}$ dans la troisième variable. Plus précisément, l'espace candidat pour faire les estimations sur $w$ peut être $\mathcal{B}_{2, \infty}^{0,-1 / 2}$, avec la norme naturelle

$$
\|w\|_{\mathcal{B}_{2, \infty}^{0,-1 / 2}}=\sup _{q}\left(2^{-\frac{q}{2}}\left\|\triangle_{q}^{\mathrm{v}} w\right\|_{L^{2}}\right) .
$$

Quand on fait les estimations d'énergie sur l'équation vérifiée par $w$ dans des espaces d'indice de régularité $-\frac{1}{2}$, on va avoir à estimer le terme $2^{-q} \int \triangle_{q}^{\mathrm{v}}\left(w^{3} \partial_{3} u\right) \triangle_{q}^{\mathrm{v}} w d x$. Sa décomposition de Bony dans la variable verticale, contient le terme

$$
I:=2^{-q} \int S_{q-1}^{\mathrm{v}} w_{3} \partial_{3} \triangle_{q}^{\mathrm{v}} u \triangle_{q}^{\mathrm{v}} w d x
$$

qui est le plus délicat à estimer. De manière générale, on va utiliser l'inégalité cidessous

$$
\int\left|S_{q-1}^{\mathrm{v}} w_{3} \partial_{3} \triangle_{q}^{\mathrm{v}} u \cdot \triangle_{q}^{\mathrm{v}} w\right| d x \leq\left\|S_{q-1}^{\mathrm{v}} w_{3}\right\|_{L_{\mathrm{v}}^{\infty} L_{h}^{2}}\left\|\triangle_{q}^{\mathrm{v}} \partial_{3} u\right\|_{L_{\mathrm{v}}^{2} L_{h}^{4}}\left\|\triangle_{q}^{\mathrm{v}} w\right\|_{L_{\mathrm{v}}^{2} L_{h}^{4}}
$$

Lorsqu'on essaie maintenant de majorer $I$ dans le cas où $w$ est estimé dans l'espace $L_{T}^{\infty}\left(\mathcal{B}_{2, \infty}^{0,-1 / 2}\right)$ avec $\nabla_{h} w$ appartenant à $L_{T}^{2}\left(\mathcal{B}_{2, \infty}^{0,-1 / 2}\right)$, on voit surgir la difficulté suivante

$$
\left\|\left(S_{q-1}^{\mathrm{v}}-S_{0}^{\mathrm{v}}\right) w_{3}\right\|_{L_{\mathrm{v}}^{\infty} L_{h}^{2}} \leq C \sum_{0 \leq q^{\prime} \leq q} 2^{q^{\prime} / 2}\left\|\triangle_{q^{\prime}}^{\mathrm{v}} w_{3}\right\|_{L^{2}} \leq C \sum_{0 \leq q^{\prime} \leq q} 2^{-q^{\prime} / 2}\left\|\triangle_{q^{\prime}}^{\mathrm{v}} \partial_{3} w_{3}\right\|_{L^{2}}
$$

En tenant compte du fait que $\partial_{3} w^{3}=-\operatorname{div}_{h} w^{h}$, on obtient

$$
\left\|\left(S_{q-1}^{\mathrm{v}}-S_{0}^{\mathrm{v}}\right) w_{3}\right\|_{L_{\mathrm{v}}^{\infty} L_{h}^{2}} \leq C q\left\|\nabla_{h} w\right\|_{\mathcal{B}_{2, \infty}^{0,-1 / 2}}
$$

L'utilisation de l'estimation (12) dans l'inégalité (11) n'est pas bénefique, elle conduit à une majoration qui ne permet pas de déduire l'unicité. Cette difficulté nous oblige de faire les estimations sur $w$ dans l'espace de Sobolev $H^{0,-1 / 2}$, afin d'abaisser la perte dans l'estimation (12). Cette fois-ci, on a l'inegalité suivante

$$
\begin{aligned}
\left\|\left(S_{q-1}^{\mathrm{v}}-S_{0}^{\mathrm{v}}\right) w_{3}\right\|_{L_{\mathrm{v}}^{\infty} L_{h}^{2}} & \leq C \sum_{0 \leq q^{\prime} \leq q} 2^{q^{\prime} / 2}\left\|\triangle_{q^{\prime}}^{\mathrm{v}} w^{3}\right\|_{L^{2}} \\
& \leq C\left(\sum_{0 \leq q^{\prime} \leq q} 2^{-q^{\prime} / 2}\left\|\triangle_{q^{\prime}}^{\mathrm{v}} \partial_{3} w_{3}\right\|_{L^{2}}\right) \\
& \leq C \sqrt{q}\left\|\nabla_{h} w\right\|_{H^{0,-1 / 2}} .
\end{aligned}
$$


Pour pouvoir utiliser cette inégalité, on introduit un paramètre $N$ et on estime différemment les "fréquences" plus petites que $N$ de celle qui sont plus grands que $N$. Pour les fréquences qui sont de taille inférieure au paramètre introduit, on utilise la régularité minimale sur $w$, cela veut dire qu'on va tenir compte du fait qu'on estime $w$ dans l'espace $H^{0,-1 / 2}$ et pour les fréquences de taille supérieure au paramètre $N$, on utilise la régularité maximale dont on dispose sur $w$, c'est-à-dire, on tient compte du fait que $w=v-u$ appartient à l'espace $H^{0,1 / 2}$ (voir aussi [17] pour cette méthode ). L'estimation sur les "hautes fréquences" conduit de manière "standard" au choix de $N$ égal à $\left(-\ln \|w\|_{H^{0,-1 / 2}}^{2}\right)$. Le problème est d'estimer les "basses fréquences". Le terme difficil est donc

$$
I_{N}:=\sum_{0 \leq q \leq N} 2^{-q} \int\left(S_{q-1}^{\mathrm{v}}-S_{0}^{\mathrm{v}}\right) w^{3} \partial_{3} \triangle_{q}^{\mathrm{v}} u \triangle_{q}^{\mathrm{v}} w d x .
$$

Ce terme pose encore des problèmes. En effet, les inégalités (13) et (11) nous conduisent à l'estimation suivante

$$
\begin{aligned}
I_{N} & \leq C \sum_{0 \leq q \leq N}\left\|\left(S_{q-1}^{\mathrm{v}}-S_{0}^{\mathrm{v}}\right) w_{3}\right\|_{L_{\mathrm{v}}^{\infty} L_{h}^{2}}\left\|\triangle_{q}^{\mathrm{v}} u\right\|_{L_{\mathrm{v}}^{2} L_{h}^{4}}\left\|\triangle_{q}^{\mathrm{v}} w\right\|_{L_{\mathrm{v}}^{2} L_{h}^{4}} \\
& \leq C \sum_{0 \leq q \leq N} \sqrt{q}\left\|\nabla_{h} w\right\|_{H^{0,-1 / 2}}\left\|\triangle_{q}^{\mathrm{v}} u\right\|_{L^{2}}^{1 / 2}\left\|\nabla_{h} \triangle_{q}^{\mathrm{v}} u\right\|_{L^{2}}^{1 / 2}\left\|\triangle_{q}^{\mathrm{v}} w\right\|_{L^{2}}^{1 / 2}\left\|\nabla_{h} \triangle_{q}^{\mathrm{v}} w\right\|_{L^{2}}^{1 / 2}
\end{aligned}
$$

En tenant compte de l'estimation

$$
\left\|\triangle_{q}^{\mathrm{v}} u\right\|_{L^{2}}^{1 / 2}\left\|\nabla_{h} \triangle_{q}^{\mathrm{v}} u\right\|_{L^{2}}^{1 / 2} \leq C 2^{-q / 2} c_{q}\|u\|_{H^{0,1 / 2}}^{1 / 2}\left\|\nabla_{h} u\right\|_{H^{0,1 / 2}}^{1 / 2}
$$

et du fait que

$$
\left\|\triangle_{q}^{\mathrm{v}} w\right\|_{L^{2}}^{1 / 2}\left\|\nabla_{h} \triangle_{q}^{\mathrm{v}} w\right\|_{L^{2}}^{1 / 2} \leq C c_{q} 2^{q / 2}\|w\|_{H^{0,-1 / 2}}^{1 / 2}\left\|\nabla_{h} w\right\|_{H^{0,-1 / 2}}^{1 / 2}
$$

on obtient finalement

$$
\begin{aligned}
I_{N} \leq \sqrt{N}\|u\|_{H^{0,1 / 2}}^{1 / 2}\left\|\nabla_{h} u\right\|_{H^{0,1 / 2}}^{1 / 2}\|w\|_{H^{0,-1 / 2}}^{1 / 2}\left\|\nabla_{h} w\right\|_{H^{0,-1 / 2}}^{3 / 2} & \\
& \leq \frac{C}{\nu_{h}} N^{2} f(t)\|w\|_{H^{0,-1 / 2}}^{2}+\frac{\nu_{h}}{10}\left\|\nabla_{h} w\right\|_{H^{0,-1 / 2}}^{2}
\end{aligned}
$$

où $f(t)=\|u(t)\|_{H^{0,1 / 2}}^{2}\left\|\nabla_{h} u(t)\right\|_{H^{0,1 / 2}}^{2}$ est fonction intégrable en temps et où le paramètre $N$ sera choisi à la fin comme étant $N=-\ln \|w\|_{H^{0,-1 / 2}}$.

Nous obtenons donc l'inégalité

$$
\frac{d}{d t}\|w(t)\|_{H^{0,-1 / 2}}^{2} \leq C f(t)\|w(t)\|_{H^{0,-1 / 2}}^{2}\left(1-\ln \|w(t)\|_{H^{0,-1 / 2}}^{2}\right)^{2},
$$

où $f(t)$ est une fonction intégrable. La perte dans cette inégalité est trop grande et ne nous permet toujours pas de déduire l'unicité. Cela provient du fait que le problème est critique en variable verticale parce qu'on a à faire des lois de produits entre des espaces avec la somme des indices de régularité nulle, mais il est également critique dans la variable horizontale parce que la régularité maximale en variable horizontale sur les fonctions utilisées est $\dot{H}^{1}\left(\mathbb{R}_{h}^{2}\right)$, espace qui n'est pas inclus dans $L^{\infty}\left(\mathbb{R}_{h}^{2}\right)$. 
Pour obtenir une estimation optimale, on introduit un nouveau paramètre $\varepsilon$ qui va quantifier le nombre des dérivées dans la variable horizontale utilisées. Le terme $I_{N}$ sera estimé maintenant de la manière qui suit. Afin d'utiliser le moins des dérivées possibles dans la variable horizontale, on utilise le lemme suivant qui est démontré dans [7]

Lemme 4.1 Il existe une constante $C$, telle qu'on a l'inéglité suivante

$$
\|f\|_{L^{2 p}\left(\mathbb{R}_{h}^{2}\right)} \leq C \sqrt{p}\|f\|_{L^{2}\left(\mathbb{R}_{h}^{2}\right)}^{1 / p}\left\|\nabla_{h} f\right\|_{L^{2}\left(\mathbb{R}_{h}^{2}\right)}^{1-1 / p} \text { pour tout } 1 \leq p<+\infty .
$$

L'inégalité de Hölder dans la variable horizontale et l'estimation (13), nous donnent

$$
\begin{aligned}
& \sum_{0 \leq q \leq N} 2^{-q} \int\left(S_{q-1}^{\mathrm{v}}-S_{0}^{\mathrm{v}}\right) w^{3} \cdot \partial_{3} \triangle_{q}^{\mathrm{v}} u \triangle_{q}^{\mathrm{v}} w d x \\
& \leq \sum_{0 \leq q \leq N} 2^{-q}\left\|\left(S_{q-1}^{\mathrm{v}}-S_{0}^{\mathrm{v}}\right) w^{3}\right\|{ }_{L_{\mathrm{v}}^{\infty} L_{h}^{2}}\left\|\partial_{3} \triangle_{q}^{\mathrm{v}} u\right\|_{L_{\mathrm{v}}^{2} L_{h}^{\frac{2}{\varepsilon}}}\left\|\triangle_{q}^{\mathrm{v}} w\right\|_{L_{\mathrm{v}}^{2} L_{h}^{\frac{2}{1-\varepsilon}}} \\
& \quad \leq \sum_{0 \leq q \leq N} C 2^{-q} \sqrt{q}\left\|\nabla_{h} w\right\|_{H^{0,-1 / 2}}\left\|\partial_{3} \triangle_{q}^{\mathrm{v}} u\right\|_{L_{\mathrm{v}}^{2} L_{h}^{\frac{2}{\varepsilon}}}\left\|\triangle_{q}^{\mathrm{v}} w\right\|_{L_{\mathrm{v}}^{2} L_{h}^{\frac{2}{1-\varepsilon}}}
\end{aligned}
$$

En utilisant maintenant le lemme 4.1 on obtient pour $0<\varepsilon<1 / 2$

$$
\begin{aligned}
& I_{N} \leq \sum_{0 \leq q \leq N} C 2^{-q} \sqrt{\frac{q}{\varepsilon}}\left\|\nabla_{h} w\right\|_{H^{0,-1 / 2}}\left\|\triangle_{q}^{\mathrm{v}} \partial_{3} u\right\|_{L^{2}}^{\varepsilon}\left\|\triangle_{q}^{\mathrm{v}} \nabla_{h} \partial_{3} u\right\|_{L^{2}}^{1-\varepsilon} \\
& \times\left\|\triangle_{q}^{\mathrm{v}} w\right\|_{L^{2}}^{1-\varepsilon}\left\|\nabla_{h} \triangle_{q}^{\mathrm{v}} w\right\|_{L^{2}}^{\varepsilon} .
\end{aligned}
$$

Vu qu'on a

$$
\left\|\triangle_{q}^{\mathrm{v}} \partial_{3} u\right\|_{L^{2}}^{\varepsilon}\left\|\nabla_{h} \triangle_{q}^{\mathrm{v}} \partial_{3} u\right\|_{L^{2}}^{1-\varepsilon} \leq 2^{q} C\left\|\triangle_{q}^{\mathrm{v}} u\right\|_{L^{2}}^{\varepsilon}\left\|\nabla_{h} \triangle_{q}^{\mathrm{v}} u\right\|_{L^{2}}^{1-\varepsilon}
$$

et en utilisant les estimations

$$
\left\|\triangle_{q}^{\mathrm{v}} u\right\|_{L^{2}}^{\varepsilon}\left\|\nabla_{h} \triangle_{q}^{\mathrm{v}} u\right\|_{L^{2}}^{1-\varepsilon} \leq 2^{-q / 2} c_{q}\|u\|_{H^{0,1 / 2}}^{\varepsilon}\left\|\nabla_{h} u\right\|_{H^{0,1 / 2}}^{1-\varepsilon}
$$

et

$$
\left\|\triangle_{q}^{\mathrm{v}} w\right\|_{L^{2}}^{1-\varepsilon}\left\|\nabla_{h} \triangle_{q}^{\mathrm{v}} w\right\|_{L^{2}}^{\varepsilon} \leq 2^{q / 2} c_{q}\|w\|_{H^{0,-1 / 2}}^{1-\varepsilon}\left\|\nabla_{h} w\right\|_{H^{0,-1 / 2}}^{\varepsilon},
$$

on obtient

$$
\begin{aligned}
\sum_{0 \leq q \leq N} 2^{-q} \int\left(S_{q-1}^{\mathrm{v}}-S_{0}^{\mathrm{v}}\right) & w^{3} \partial_{3} \triangle_{q}^{\mathrm{v}} u \triangle_{q}^{\mathrm{v}} w d x \\
& \leq C \sqrt{\frac{N}{\varepsilon}}\|u\|_{H^{0,1 / 2}}^{\varepsilon}\left\|\nabla_{h} u\right\|_{H^{0,1 / 2}}^{1-\varepsilon}\|w\|_{H^{0,-1 / 2}}^{1-\varepsilon}\left\|\nabla_{h} w\right\|_{H^{0,-1 / 2}}^{1+\varepsilon}
\end{aligned}
$$

En utilisant maintenant l'inégalité $a b \leq a^{\frac{2}{1+\varepsilon}}+b^{\frac{2}{1-\varepsilon}}$, on obtient

$$
I_{N} \leq C\left(\frac{N}{\varepsilon}\right)^{\frac{1}{1-\varepsilon}}\|u\|_{H^{0,1 / 2}}^{\frac{2 \varepsilon}{1-\varepsilon}}\left\|\nabla_{h} u\right\|_{H^{0,1 / 2}}^{2}\|w\|_{H^{0,-1 / 2}}^{2}+\frac{\nu_{h}}{2}\left\|\nabla_{h} w\right\|_{H^{0,-1 / 2}}^{2}
$$


Pour optimiser l'estimation, on fait le choix de $\varepsilon$ égal à $\frac{1}{1+\ln N}$ et on obtient

$$
\begin{aligned}
\sum_{0 \leq q \leq N} 2^{-q} \int\left(S_{q-1}^{\mathrm{v}}-S_{0}^{\mathrm{v}}\right) w^{3} \partial_{3}^{\mathrm{v}} \triangle_{q}^{\mathrm{v}} u \triangle_{q}^{\mathrm{v}} w d x & \\
& \leq C N(1+\ln N) f(t)\|w\|_{H^{0,-1 / 2}}^{2}+\frac{\nu_{h}}{2}\left\|\nabla_{h} w\right\|_{H^{0,-1 / 2}}^{2}
\end{aligned}
$$

Le choix de $N=-\ln \|w\|_{H^{0,-1 / 2}}^{2}$, nous amène à l'inégalité différentielle

$$
\frac{d}{d t}\|w(t)\|_{H^{0,-1 / 2}}^{2} \leq C\|w(t)\|_{H^{0,-1 / 2}}^{2}\left(-\ln \|w(t)\|_{H^{0,-1 / 2}}^{2}\right) \ln \left(-\ln \|w(t)\|_{H^{0,-1 / 2}}^{2}\right) .
$$

Expliquons maintenant le choix de $N$. Pour aboutir à une estimation sur

$$
\sum_{q} 2^{-q} \int S_{q-1}^{\mathrm{v}} w^{3} \partial_{3} \triangle_{q}^{\mathrm{v}} u \triangle_{q}^{\mathrm{v}} w d x,
$$

il est necessaire d'éstimer aussi la somme sur les fréquences supérieures au paramètre $N$. On a

$$
\begin{aligned}
& I^{N}:=\sum_{q} 2^{-q} \int \sum_{j \geq N} \triangle_{j}^{\mathrm{v}} w^{3} \partial_{3} \triangle_{q}^{\mathrm{v}} u \triangle_{q}^{\mathrm{v}} w d x \\
& \quad \leq C \sum_{q} \sum_{j \geq N}\left\|\triangle_{j}^{\mathrm{v}} w^{3}\right\|_{L_{\mathrm{v}}^{\infty} L_{h}^{2}}\left\|\triangle_{q}^{\mathrm{v}} u\right\|_{L_{\mathrm{v}}^{2} L_{h}^{4}}\left\|\triangle_{q}^{\mathrm{v}} w\right\|_{L_{\mathrm{v}}^{2} L_{h}^{4}} .
\end{aligned}
$$

D'autre part, en utilisant le fait que $\nabla_{h}$ w appartient à l'espace $H^{0,1 / 2}$, on a

$$
\begin{aligned}
\sum_{j \geq N}\left\|\triangle_{q}^{\mathrm{v}} w^{3}\right\|_{L_{\mathrm{v}}^{\infty} L_{h}^{2}} \leq C \sum_{j \geq N} 2^{-j / 2}\left\|\triangle_{j}^{\mathrm{v}} \nabla_{h} w\right\|_{L^{2}} & \leq C \sum_{j \geq N} C 2^{-j} c_{j}\left\|\nabla_{h} w\right\|_{H^{0,1 / 2}} \\
& \leq C 2^{-N}\left\|\nabla_{h} w\right\|_{H^{0,1 / 2}}
\end{aligned}
$$

En utilisant (17) dans l'inégalité (16), on obtient

$$
\begin{aligned}
I^{N} & \leq C 2^{-N}\left\|\nabla_{h} w\right\|_{H^{0,1 / 2}} \sum_{q}\left\|\triangle_{q}^{\mathrm{v}} u\right\|_{L^{2}}^{1 / 2}\left\|\nabla_{h} \triangle_{q}^{\mathrm{v}} u\right\|_{L^{2}}^{1 / 2}\left\|\triangle_{q}^{\mathrm{v}} w\right\|_{L^{2}}^{1 / 2}\left\|\nabla_{h} \triangle_{q}^{\mathrm{v}} w\right\|_{L^{2}}^{1 / 2} \\
& \leq C 2^{-N}\left\|\nabla_{h} w\right\|_{H^{0,1 / 2}}\|u\|_{H^{0,1 / 2}}^{1 / 2}\left\|\nabla_{h} u\right\|_{H^{0,1 / 2}}^{1 / 2}\|w\|_{H^{0,-1 / 2}}^{1 / 2}\left\|\nabla_{h} w\right\|_{H^{0,-1 / 2}}^{1 / 2} .
\end{aligned}
$$

En utilisant l'inégalité $x y \leq \frac{3}{4} x^{4 / 3}+\frac{1}{4} y^{4}$, on obtient

$$
\begin{array}{r}
I^{N} \leq C 2^{-4 N / 3}\left\|\nabla_{h} w\right\|_{H^{0,1 / 2}}^{4 / 3}\|u\|_{H^{0,1 / 2}}^{2 / 3}\left\|\nabla_{h} u\right\|_{H^{0,1 / 2}}^{2 / 3}\|w\|_{H^{0,-1 / 2}}^{2 / 3}+\frac{\nu_{h}}{10}\left\|\nabla_{h} w\right\|_{H^{0,-1 / 2}}^{2} \\
\leq C 2^{-4 N / 3} f(t)\|w\|_{H^{0,-1 / 2}}^{2 / 3}+\frac{\nu_{h}}{10}\left\|\nabla_{h} w\right\|_{H^{0,-1 / 2}}^{2} .
\end{array}
$$

Afin de pouvoir utiliser sur ce terme le lemme de Gronwall, il faut prendre $N$ dans l'estimation ci-avant, tel que

$$
2^{-4 N / 3} C f(t)\|w\|_{H^{0,-1 / 2}}^{2 / 3}=C f(t)\|w(t)\|_{H^{0,-1 / 2}}^{2} .
$$


On obtient ainsi à une constante près $N=1-\ln \|w(t)\|_{H^{0,-1 / 2}}^{2}$.

La démostration complète de l'estimation (15) est donnée dans l'article [14]. Par consequent, nous n'allons pas donner ici les autres termes à estimer. Remarquons seulement que la démontration de l'unicité dans l'espace $\mathcal{B}^{0,1 / 2}$ est plus simple que la preuve de l'unicité dans l'espace $H^{0,1 / 2}$, puisqu'on a $\left\|S_{q}^{\mathrm{v}} u\right\|_{L_{\mathrm{v}}^{\infty}\left(L_{h}^{2}\right)} \leq C \|_{u \|_{\mathcal{B}^{0,1 / 2}} \text {, }}$ or dans l'espace $H^{0,1 / 2}$ on a à eviter la difficulté $\left\|S_{q}^{\mathrm{v}} u\right\|_{L_{\mathrm{v}}^{\infty}\left(L_{h}^{2}\right)} \leq C \sqrt{q}\|u\|_{H^{0,1 / 2}}$.

La fin de la démonstration de l'unicité, est une application directe du lemme 3.2. L'équation vérifiée par $w$ est la suivante

$$
\partial_{t} w+v \nabla w+w \nabla u-\nu_{h} \Delta_{h} w=-\nabla p .
$$

Le lemme 3.2, nous donne l'estimation

$$
\frac{d}{d t}\|w(t)\|_{H^{0,-1 / 2}}^{2} \leq C f(t)\|w(t)\|_{H^{0,-1 / 2}}^{2}\left(1-\ln \|w(t)\|_{H^{0,-1 / 2}}^{2}\right) \ln \left(1-\ln \|w(t)\|_{H^{0,-1 / 2}}^{2}\right)
$$

où $f(t)$ est une fonction intégrable sur l'intervalle $[0, T]$ avec

$$
\begin{aligned}
\|f\|_{L_{T}^{1}} \leq C_{T}(1+ & \left.\left\|\nabla_{h} u\right\|_{L_{T}^{2}\left(H^{0,1 / 2}\right)}+\left\|\nabla_{h} v\right\|_{L_{T}^{2}\left(H^{0,1 / 2}\right)}+\left\|\nabla_{h} w\right\|_{L_{T}^{2}\left(H^{0,1 / 2}\right)}\right) \\
& \times\left(1+\|u\|_{L_{T}^{\infty}\left(H^{0,1 / 2}\right)}+\|v\|_{L_{T}^{\infty}\left(H^{0,1 / 2}\right)}+\|w\|_{L_{T}^{\infty}\left(H^{0,1 / 2}\right)}\right) .
\end{aligned}
$$

En tenant compte du fait que $\|w\|_{L_{T}^{\infty}\left(H^{0,1 / 2}\right)} \leq\|u\|_{L_{T}^{\infty}\left(H^{0,1 / 2}\right)}+\|v\|_{L_{T}^{\infty}\left(H^{0,1 / 2}\right)}$, et aussi $\left\|\nabla_{h} w\right\|_{L_{T}^{2}\left(H^{0,1 / 2}\right)} \leq\left\|\nabla_{h} u\right\|_{L_{T}^{2}\left(H^{0,1 / 2}\right)}+\left\|\nabla_{h} v\right\|_{L_{T}^{2}\left(H^{0,1 / 2}\right)}$, on obtient donc dans notre cas, le fait que $f$ est fonction localement intégrable.

Rappellons à présent le lemme d'Osgood (voir le livre [8] ou l'article [5]), qui va nous permettre de conclure l'unicité.

\section{Lemme 4.2 (Osgood)}

Soient $\rho \geq 0$ une fonction mesurable, $\gamma$ une fonction localement intégrable et $\mu$ une fonction continue croissante qui vérifie la condition

$$
\int_{0}^{1} \frac{d r}{\mu(r)}=+\infty
$$

Soit aussi a un nombre réel positif. Si $\rho$ vérifie l'inégalité

$$
\rho(t) \leq a+\int_{0}^{t} \gamma(s) \mu(\rho(s)) d s .
$$

Alors, si a est nul la fonction $\rho$ est identiquement nulle. Si a est non nul, alors on $a$

$$
-\mathcal{M}(\rho(t))+\mathcal{M}(a) \leq \int_{0}^{t} \gamma(s) d s, \quad \text { avec } \quad \mathcal{M}(x)=\int_{x}^{1} \frac{d r}{\mu(r)} .
$$

Finalement, l'application du lemme d'Osgood, dans le cas où on choisi $\rho(s):=$ $\|w(s)\|_{H^{0,-1 / 2}}^{2}$ et $\mu(r):=r(1-\ln r) \ln (1-\ln r)$, implique l'unicité. 


\section{Références}

[1] J.-M. Bony, Calcul symbolique et propagation des singularités pour les équations aux dérivées partielles non linéaires, Annales scientifiques de l'École Normale Supérieure, 14, 209-246, 1981.

[2] M. Cannone, Ondelettes, paraproduits et Navier-Stokes, Nouveaux essais, Diderot éditeurs, 1995.

[3] J.-Y. Chemin, Fluides Parfaits Incompressibles. Astérisque, 230, 1995.

[4] J.-Y. Chemin, Théorèmes d'unicité pour le système de Navier-Stokes tridimensionel, Journal d'Analyse Mathématique, 77, p. 27-50, 1999.

[5] J.-Y. Chemin et N. Lerner, Flot de champs de vecteurs non Lipschitziens et équations de Navier-Stokes, Journal of Differential Equations, 121, p. 314-328, 1992.

[6] J.-Y. Chemin, B. Desjardins, I. Gallagher et E. Grenier, Fluids with anisotropic viscosity, M2AN. Math. Numer. Anal., 34, No. 2, p. 315-335, 2000.

[7] J.-Y. Chemin, C.-J. Xu, Inclusions de Sobolev en calcul de Weyl-Hormander et champs de vecteurs sous-elliptiques, Ann. Sci. École Norm. Sup. (4) 30 (1997), no. 6, 719-751.

[8] T. M. Fleet, Differential analysis, Cambridge University Press, 1980.

[9] H. Fujita et T. Kato, On the Navier-Stokes initial value problem I, Archiv for Rational Mechanic and Analysis, 16, p. 269-315, 1964.

[10] E. Grenier, N. Masmoudi, Ekman layers of rotating fluid, the case of well prepared initial data, Comm. Partial Differential Equations, 22, no. 5-6, p. 953975, 1997.

[11] D. Iftimie, Resolution of the Navier-Stokes equations in anisotropic spaces, Revista Matématica Iberoamericana, 15, no. 1, 1-36, 1999.

[12] D. Iftimie, A Uniqueness result for the Navier-Stokes Equations with vanishing vertical viscosity, à paraître dans SIAM J. Math. Analysis.

[13] J. Leray, Sur le mouvement d'un liquide visqueux remplissant l'espace, Acta Math, 63, p. 193-248, 1934.

[14] M. Paicu, Équation de Navier-Stokes anisotropes dans des espaces critiques, à paraître dans Revista Matématica Iberoamericana.

[15] J. Pedlosky, Geophysical Fluid Dynamics, Springer Verlag, New York, 1987.

[16] F. Planchon, Global strong solutions in Sobolev or Lebesgue spaces to the incompressible Navier-Stokes equations in $\mathbb{R}^{3}$, Annales de l'Institut Henri Poincaré, 13, no. 3, 319-336, 1996.

[17] M. Vishik, Incompressible flows of an ideal fluid with vorticity in borderline spaces of Besov type, Ann. Sci. École Norm. Sup. (4) 32 (1999), no. 6, 769-812.

Centre de Mathématiques de l'École Polytechnique, UMR 7640 CNRS, 91128 Palaiseau Cedex, France.

paicu@math. polytechnique.fr 